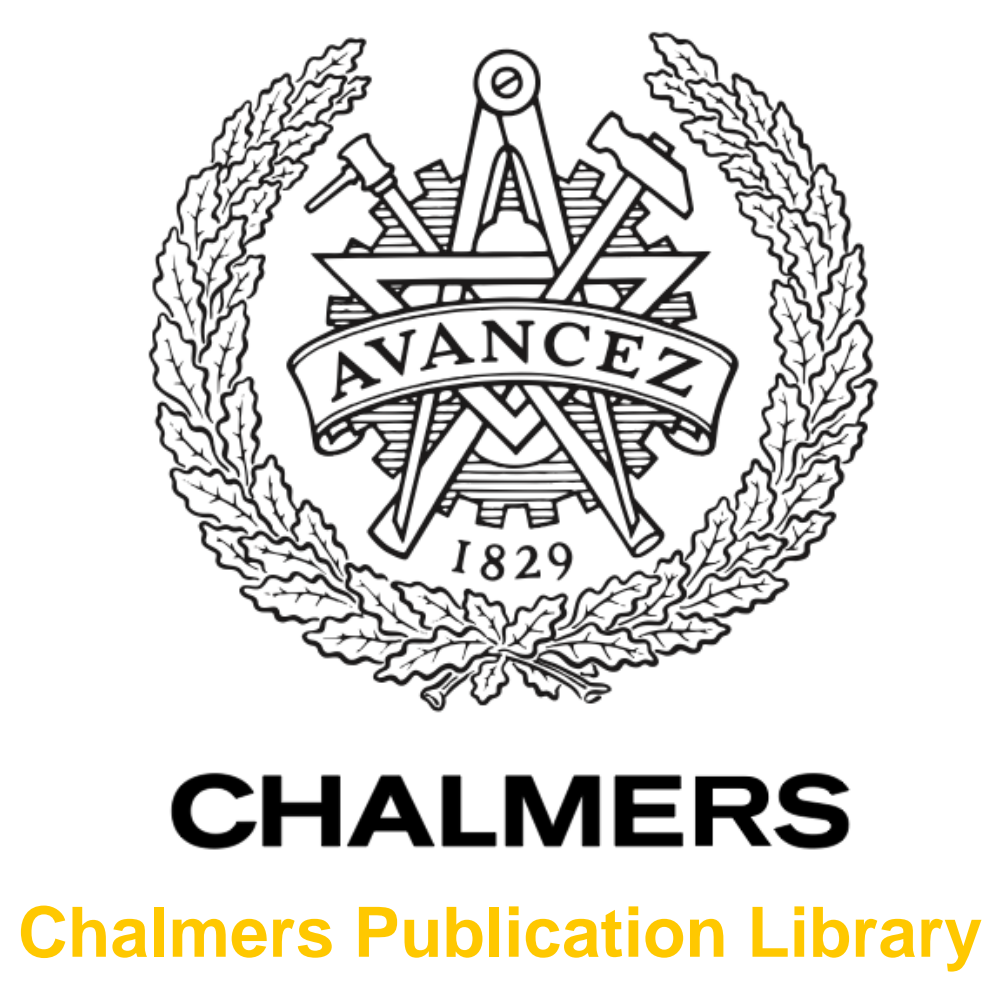

\title{
The propagation of in-plane P-SV waves in a layered elastic plate with periodic interface cracks: exact versus spring boundary conditions
}

This document has been downloaded from Chalmers Publication Library (CPL). It is the author's version of a work that was accepted for publication in:

Waves in Random and Complex Media (ISSN: 1745-5030)

\author{
Citation for the published paper: \\ Kvasha, O. ; Boström, A. ; Glushkova, N. et al. (2011) "The propagation of in-plane P-SV \\ waves in a layered elastic plate with periodic interface cracks: exact versus spring boundary \\ conditions". Waves in Random and Complex Media, vol. 21(3), pp. 515-528.
}

http://dx.doi.org/10.1080/17455030.2011.593586

Downloaded from: http://publications.lib.chalmers.se/publication/156538

Notice: Changes introduced as a result of publishing processes such as copy-editing and formatting may not be reflected in this document. For a definitive version of this work, please refer to the published source. Please note that access to the published version might require a subscription. 


\title{
RESEARCH ARTICLE
}

\section{The propagation of in-plane $P-S V$ waves in a layered elastic plate with periodic interface cracks: exact versus spring boundary conditions}

\author{
Oleg V. Kvasha ${ }^{\mathrm{a}}$ (deceased), Anders Boström ${ }^{\mathrm{b} *}$, Natalia V. Glushkova ${ }^{\mathrm{a}}$, and Evgeny V. \\ Glushkov $^{\mathrm{a}}$ \\ anstitute for Mathematics, Mechanics and Informatics, Kuban State University, \\ Krasnodar 350040, Russia; ${ }^{\mathrm{b}}$ Department of Applied Mechanics, Chalmers University of \\ Technology, SE-412 96 Göteborg, Sweden
}

( February 2011)

\begin{abstract}
The propagation of in-plane (P-SV) waves in a symmetrically three-layered thick plate with a periodic array of interface cracks are investigated. The exact dispersion relation is derived based on an integral equation approach and Floquet's theorem. The interface cracks can be a model for interface damage, but a much simpler model is a recently developed spring boundary condition. This boundary condition is used for the thick plate and also in the derivation of plate equations with the help of power series expansions in the thickness coordinate. For low frequencies (cracks small compared to the wavelength) the three approaches give more or less coinciding dispersion curves, and this is a confirmation that the spring boundary condition is a reasonable approximation at low frequencies.
\end{abstract}

Keywords: elastic waves; periodic cracks; spring boundary condition; plate equations

\section{Introduction}

Damage in a layered composite often occurs at the interfaces and this can be both as a total delamination or as a more diffuse damage in the form of micro-cracks, small voids, etc. The latter type of damage is not so easily modelled in the context of ultrasonic wave propagation and nondestructive testing. One way is to model the damage with a spring boundary condition, see Golub and Boström [1], where the spring constant is derived for a random distribution of small cracks between two isotropic media. Another way is to model the damage as a periodic set of small interface cracks, see Boström and Kvasha [2] for the 2D anti-plane (SH) case. In the present paper this approach is applied to the in-plane (P-SV) case. Elastic wave propagation in a layered plate with interface damage in the form of a periodic distribution of cracks is compared with the corresponding plate with spring boundary conditions between the layers. Furthermore, a comparison with a plate equation incorporating the spring boundary conditions is also performed.

A general good introduction to the elastic wave propagation and scattering in the presence of cracks is given by Zhang and Gross [3], which in particular also investigate a periodic distribution of cracks. Angel and Achenbach [4] and Mikata and Achenbach [5] consider a periodic distribution of interface cracks between two

*Corresponding author. Email: anders.bostrom@chalmers.se 
isotropic half-spaces. Spring boundary conditions have been employed to model different types of interfaces: Baik and Thompson [6], Rokhlin and Wang [7], and Rokhlin and Huang [8] model various types of imperfect interfaces, while Boström and Wickham [9], Nakagawa et al. [10], and Pecorari [11] model partially closed cracks. Layers of damaged material are considered by Achenbach and Zhang [12] and Achenbach et al. [13].

The plan of the present paper is as follows. In Section 2 the problem for the in-plane modes in a symmetrically three-layered thick plate is formulated, and in Section 3 the solution and the dispersion relation are derived with the help of an integral equation approach and Floquet's (or Bloch's) theorem. In Section 4 the spring boundary condition is stated and the symmetric (in-plane) and antisymmetric (bending) plate equations are derived. In Section 5 the dispersion relations for the three approaches are compared and Section 6 offers a few concluding remarks.

\section{Formulation of the problem}

Consider a symmetrically three-layered isotropic thick plate according to Fig. 1. This geometry is chosen because it is simple and admits a subdivision into symmetric and antisymmetric parts. However, in principle any configuration of layers can be chosen. The middle layer has thickness $2 d_{1}$ and the two outer layers have thickness $d_{2}-d_{1}$ each; the total thickness of the plate is $d=2 d_{2}$. The material parameters in the layers are denoted by the index $j=1,2$ for the middle and outer layers, respectively. Thus, $\lambda_{j}, \mu_{j}$ and $\rho_{j}$ are the Lamé constants and densities of the middle $(j=1)$ and outer $(j=2)$ layers. All the cracks are of equal width $2 l$, periodically located with centers at $x=m a, z= \pm d_{1}, m=0, \pm 1, \pm 2, \ldots, a>2 l$.

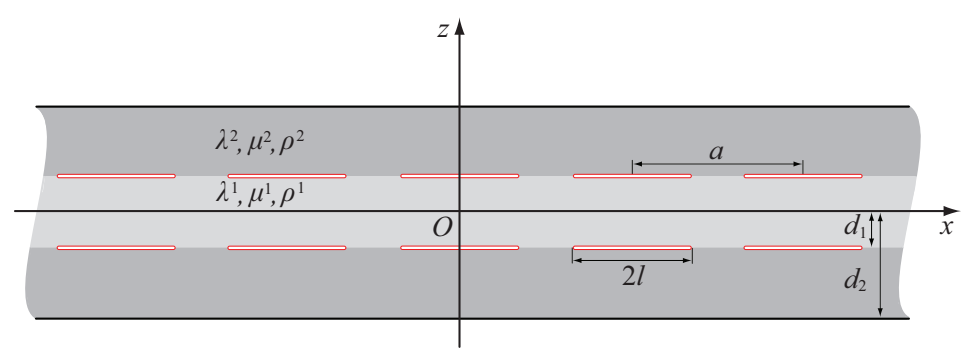

Figure 1. Elastic plate with periodic arrays of cracks.

In-plane coupled compressional and shear (P-SV) wave propagation is considered. Time harmonic waves are assumed, with the time factor $e^{-i \omega t}$ suppressed throughout. The displacement vectors $\mathbf{u}^{j}=\left(u_{x}^{j}, u_{z}^{j}\right)^{T}$ obey the Lamé equations

$$
\left(\lambda_{j}+\mu_{j}\right) \nabla \nabla \cdot \mathbf{u}^{j}+\mu_{j} \Delta \mathbf{u}^{j}+\rho_{j} \omega^{2} \mathbf{u}^{j}=0, \quad j=1,2 .
$$

The three relevant stress components are

$$
\begin{gathered}
\sigma_{x}^{j}=\left(\lambda_{j}+2 \mu_{j}\right) \frac{\partial u_{x}^{j}}{\partial x}+\lambda_{j} \frac{\partial u_{z}^{j}}{\partial z}, \\
\sigma_{z}^{j}=\lambda_{j} \frac{\partial u_{x}^{j}}{\partial x}+\left(\lambda_{j}+2 \mu_{j}\right) \frac{\partial u_{z}^{j}}{\partial z},
\end{gathered}
$$


and

$$
\tau_{x z}^{j}=\mu_{j}\left(\frac{\partial u_{x}^{j}}{\partial z}+\frac{\partial u_{z}^{j}}{\partial x}\right), \quad j=1,2
$$

From the symmetry it follows that only one half of the plate $\left(-d_{2}<z<0\right)$ needs to be considered and that the problem can be decomposed into symmetric and antisymmetric parts. The boundary conditions for the antisymmetric part are

$$
\left.u_{x}^{1}\right|_{z=0}=0,\left.\quad \sigma_{z}^{1}\right|_{z=0}=0 \quad \text { and }\left.\quad \sigma^{2}\right|_{z=-d_{2}}=0 .
$$

For the symmetric part they are instead

$$
\left.u_{z}^{1}\right|_{z=0}=0,\left.\quad \tau_{x z}^{1}\right|_{z=0}=0 \quad \text { and }\left.\quad \boldsymbol{\sigma}^{2}\right|_{z=-d_{2}}=0 .
$$

Here $\boldsymbol{\sigma}^{j}=\left(\tau_{x z}^{j}, \sigma_{z}^{j}\right)^{T}$ is the stress vector at a horizontal surface element. On the interface $z=-d_{1}$ the displacement and traction are continuous except on the cracks, where the boundary conditions are

$$
\left.\boldsymbol{\sigma}^{1}\right|_{z=-d_{1}}=\left.\boldsymbol{\sigma}^{2}\right|_{z=-d_{1}}=0, \quad|x-m a|<l, \quad m=0, \pm 1, \pm 2, \ldots
$$

This gives rise to a displacement discontinuity on the cracks with the crack-opening displacements (c.o.d.) defined as

$$
\mathbf{v}_{m}(x)=\mathbf{u}^{1}\left(x,-d_{1}\right)-\mathbf{u}^{2}\left(x,-d_{1}\right), \quad|x-m a|<l, \quad m=0, \pm 1, \pm 2, \ldots .
$$

As the plate with the cracks is a periodic system, the Bloch-Floquet theorem [14] states that the modes in the plate have the property

$$
\mathbf{u}^{j}(x, z)=e^{i k x} \mathbf{w}^{j}(x, z), \quad j=1,2,
$$

where $\mathbf{w}^{j}(x, z)$ are periodic functions with the period $a$ :

$$
\mathbf{w}^{j}(x+m a, z)=\mathbf{w}^{j}(x, z), \quad m=0, \pm 1, \pm 2, \ldots, \quad j=1,2 .
$$

The main goal here is to find the dispersion relation $k=k(\omega)$ for non-zero solutions (5) obeying Eq. (1), homogeneous boundary conditions (2) or (3), the conditions of stress and displacement continuity at the interface $z=-d_{1}$ outside the cracks, and the zero-traction condition (4) on the crack.

\section{Exact solution}

In this section the exact dispersion relation is derived. This is done by representing the fields by Fourier transforms with Green's functions, which upon using the boundary conditions and the Bloch-Floquet theorem lead to an integral equation on a single crack. This equation is discretized by an expansion in Chebyshev polynomials with a square-root weight to account for the singularity at the crack tips. An alternative, essentially equivalent, way would be to use a Fourier series representation over a single cell which together with the Bloch-Floquet theorem should lead to the same integral equation. 
To derive a general solution the Fourier transform $F_{x}$ :

$$
F_{x}\left[\mathbf{u}^{j}\right]=\int_{-\infty}^{\infty} \mathbf{u}^{j}(x, z) e^{i \alpha x} d x=\mathbf{U}^{j}(\alpha, z), \quad j=1,2
$$

is applied to the equations and boundary conditions of the problem considered. In this way, the wave fields $\mathbf{u}^{j}$ are sought for in the form of the inverse Fourier transform:

$$
\mathbf{u}^{j}(x, z)=F_{x}^{-1}\left[\mathbf{U}^{j}\right]=\frac{1}{2 \pi} \int_{\Gamma} \mathbf{U}^{j}(\alpha, z) e^{-i \alpha x} d \alpha, \quad j=1,2 .
$$

The integration path $\Gamma$ goes in the complex $\alpha$ plane along the real axis $\operatorname{Im} \alpha=0$, deviating from it for bypassing real singularities of the integrands in accordance with the principle of limiting absorption [15]. In the Fourier transform domain the solution $\mathbf{U}^{j}$ is obtained in the form

$$
\mathbf{U}^{j}(\alpha, z)=K^{j}(\alpha, z) \mathbf{Q}(\alpha), \quad j=1,2,
$$

where $K^{j}=F_{x}\left[k^{j}\right]$ are the Fourier transforms of the Green's matrices $k^{j}(x, z)$ for the layers $-d_{1}<z<0(j=1)$ and $-d_{2}<z<d_{1}(j=2)$ and $\mathbf{Q}=F_{x}[\mathbf{q}]$ is the transform of an unknown stress $\mathbf{q}(x)$ at the contact interface $z=-d_{1}$.

Mathematically, the columns $\mathbf{k}_{n}^{1}(x, z), n=1,2$ of the two-by-two matrix $k^{1}(x, z)$ are the solutions to Eq. (1) with $j=1$ satisfying the boundary conditions

$$
\left.u_{x}^{1}\right|_{z=0}=0,\left.\quad \sigma_{z}^{1}\right|_{z=0}=0 \quad \text { and }\left.\quad \boldsymbol{\sigma}^{1}\right|_{z=-d_{1}}=\delta(x) \mathbf{e}_{n}
$$

or

$$
\left.u_{z}^{1}\right|_{z=0}=0,\left.\quad \tau_{x z}^{1}\right|_{z=0}=0 \quad \text { and }\left.\quad \boldsymbol{\sigma}^{1}\right|_{z=-d_{1}}=\delta(x) \mathbf{e}_{n}
$$

where $\mathbf{e}_{1}=(1,0)^{T}$ and $\mathbf{e}_{2}=(0,1)^{T}$ are the unit coordinate vectors and $\delta(x)$ is Dirac's delta function. As in Eqs. (2) and (3), the choice of the boundary conditions depends on whether the antisymmetric or the symmetric solution is considered. Analogously, the columns $\mathbf{k}_{n}^{2}, n=1,2$, of the matrix $k^{2}(x, z)$ obey Eq. (1) with $j=2$ and the boundary conditions

$$
\left.\boldsymbol{\sigma}^{2}\right|_{z=-d_{1}}=\delta(x) \mathbf{e}_{n} \quad \text { and }\left.\quad \boldsymbol{\sigma}^{2}\right|_{z=-d_{2}}=0 .
$$

The matrices $K^{j}(\alpha, z)$ for homogeneous elastic layers are derived in a closed form (e.g. [16]).

Eq. (7) implies a connection between the unknown vector functions $\mathbf{q}(x)$ and $\mathbf{v}_{m}(x)$ set at the interface. Indeed, taking the difference of the transforms $\mathbf{U}^{j}$ specified by Eq. (7) yields at $z=-d_{1}$

$$
\mathbf{U}^{1}\left(\alpha,-d_{1}\right)-\mathbf{U}^{2}\left(\alpha,-d_{1}\right)=\left[K^{1}\left(\alpha,-d_{1}\right)-K^{2}\left(\alpha,-d_{1}\right)\right] \mathbf{Q}(\alpha)=\sum_{m=-\infty}^{\infty} \mathbf{V}_{m}(\alpha)
$$


where $\mathbf{V}_{m}=F_{x}\left[\mathbf{v}_{m}\right]$. Hence,

$$
\mathbf{Q}(\alpha)=L(\alpha) \sum_{m=-\infty}^{\infty} \mathbf{V}_{m}(\alpha), \quad L(\alpha)=\left[K^{1}\left(\alpha,-d_{1}\right)-K^{2}\left(\alpha,-d_{1}\right)\right]^{-1}
$$

Furthermore, Eq. (7) can be rewritten in terms of $\mathbf{V}_{m}$ :

$$
\mathbf{U}^{j}(\alpha, z)=N^{j}(\alpha, z) \sum_{m=-\infty}^{\infty} \mathbf{V}_{m}(\alpha), \quad N^{j}(\alpha, z)=K^{j}(\alpha, z) L(\alpha), \quad j=1,2,
$$

which leads to the integral representations

$$
\mathbf{u}^{j}(x, z)=\frac{1}{2 \pi} \int_{\Gamma} N^{j}(\alpha, z) \sum_{m=-\infty}^{\infty} \mathbf{V}_{m}(\alpha) e^{-i \alpha x} d \alpha, \quad j=1,2
$$

Due to the periodicity property specified by Eq. (5), every function $\mathbf{V}_{m}$ can be expressed via the single function $\mathbf{V}_{0}$ :

$\mathbf{V}_{m}(\alpha)=\int_{m a-l}^{m a+l} \mathbf{v}_{m}(x) e^{i \alpha x} d x=\int_{-l}^{l} \mathbf{v}_{0}(x) e^{i(\alpha x+(\alpha+k) m a)} d x=\mathbf{V}_{0}(\alpha) e^{i(\alpha+k) m a}, \quad m=0, \pm 1, \pm 2, \ldots$

The substitution of $\mathbf{V}_{m}(\alpha)$ in this form into Eq. (10) and the use of the formula $[3]$

$$
\sum_{m=-\infty}^{\infty} e^{i(\alpha+k) m a}=2 \pi \sum_{m=-\infty}^{\infty} \delta\left(\frac{\alpha+k}{2 \pi} a-m\right)
$$

lead to a closed-form series representation for the periodic functions $\mathbf{w}^{j}$ in Eq. (5):

$\mathbf{w}^{j}(x, z)=\frac{1}{a} \sum_{m=-\infty}^{\infty} N^{j}\left(\alpha_{m}, z\right) \mathbf{V}_{0}\left(\alpha_{m}\right) e^{-i \beta_{m} x}, \quad \beta_{m}=2 \pi m / a, \quad \alpha_{m}=\beta_{m}-k, \quad j=1,2$.

In the same way, Eq. (9) yields $\mathbf{q}=F_{x}^{-1}[\mathbf{Q}]$ in the closed series form

$$
\mathbf{q}(x)=\frac{1}{a} \sum_{m=-\infty}^{\infty} \mathbf{L}\left(\alpha_{m}, z\right) \mathbf{V}_{0}\left(\alpha_{m}\right) e^{-i \alpha_{m} x}
$$

Since the crack sides are stress-free (see Eq. (4)), $\mathbf{q}=0$ at the segment $|x|<l$. In fact, this condition reduces the problem to the homogeneous integral equation with respect to the c.o.d. $\mathbf{v}_{0}(x)$ :

$$
\sum_{m=-\infty}^{\infty} L\left(\alpha_{m}\right) \mathbf{V}_{0}\left(\alpha_{m}\right) e^{-i \alpha_{m} x}=0, \quad|x|<l
$$




$$
\mathbf{V}_{0}(\alpha)=F_{x}\left[\mathbf{v}_{0}\right]=\int_{-l}^{l} \mathbf{v}_{0}(x) e^{i \alpha x} d x
$$

The values $k(\omega)$, for which non-trivial solutions to Eq. (13) exist, are the wave numbers of modes (5), propagating in the plate at a given frequency $\omega$, while Eq. (11) to constant multipliers yields their spatial eigenforms. In the latter, the vectors $\mathbf{V}_{0}\left(\alpha_{m}\right)$ are expressed via the eigensolutions $\mathbf{v}_{0}(x)$ associated with the roots $k=k(\omega)$.

To discretize Eq. (13), the c.o.d. $\mathbf{v}_{0}$ is expanded in terms of the Chebyshev polynomials of the second kind $U_{n}(x)$ with square-root weight:

$$
\mathbf{v}_{0}(x)=\sum_{n=0}^{\infty} \mathbf{c}_{n} p_{n}(x), \quad p_{n}(x)=U_{n}(x / l) \sqrt{1-(x / l)^{2}} .
$$

The weight takes care of the square root singularity at the crack tips, but the oscillating factor that is generally also present in the case of two different materials is not included into Eq. (14). Nevertheless, it is enough to assure numerical stability of the calculations below. The substitution of expansion (14) into Eq. (13) yields

$$
\sum_{n=0}^{\infty}\left[\frac{1}{a} \sum_{m=-\infty}^{\infty} L\left(\alpha_{m}\right) P_{n}\left(\alpha_{m}\right) e^{-i \alpha_{m} x}\right] \mathbf{c}_{n}=0, \quad|x|<l,
$$

where

$$
P_{n}(\alpha)=F_{x}\left[p_{n}\right]=\pi l i^{n}(n+1) J_{n+1}(\alpha l) / \alpha
$$

and $J_{n+1}(\alpha l)$ are the Bessel functions. Following the Galerkin scheme, Eq. (15) is multiplied by $p_{j}(x)$ for $j=0,1,2, \ldots$ and integrated over $-l<x<l$. This reduces Eq. (15) to an infinite homogeneous system of linear algebraic equations with respect to the vector of expansion coefficients $\mathbf{c}=\left(\mathbf{c}_{1}, \mathbf{c}_{2}, \ldots, \mathbf{c}_{n}, \ldots\right)^{T}$ :

$$
A(k, \omega) \mathbf{c}=0
$$

The matrix $A=\left[A_{j n}\right]_{0}^{\infty}$ consists of $2 \times 2$ blocks

$$
A_{j n}(k, \omega)=\frac{1}{a} \sum_{m=-\infty}^{\infty} L\left(\alpha_{m}\right) P_{n}\left(\alpha_{m}\right) P_{j}\left(-\alpha_{m}\right), \quad j, n=0,1,2, \ldots
$$

Non-trivial solutions to system (16) exist when the matrix $A(k, \omega)$ becomes singular, that is

$$
\operatorname{det}(A(k, \omega))=0
$$

Eq. (18) is the sought-for dispersion relation. It implicitly relates the wave number $k$ to the frequency $\omega$. With the series representations (13), (14) dispersion equation (18) is explicit, but for numerical calculations the series, obviously, must be truncated. The number of terms in the truncated series is determined from numerical experiments to meet the accuracy required for the roots $k=k(\omega)$. 


\section{Approximate plate equations}

If the cracks are small as compared to characteristic wavelengths and plate dimensions, it is possible to consider the layered plate in a simple fashion using an approximate spring boundary condition between the layers. First, it is noted that the exact distribution of small cracks is expected to be unimportant. For both the SH and the P-SV cases Boström and Golub [17] and Golub and Boström [1] have derived an approximate spring boundary condition to model an interface with a random distribution of small cracks of the same size. This boundary condition should also be applicable to the periodic distribution of interface cracks in the plate considered. Thus, the exact periodic boundary conditions at the interface $z=-d_{1}$ are replaced by the simplified spring boundary condition:

$$
\boldsymbol{\sigma}^{1}=\boldsymbol{\sigma}^{2}=\kappa\left(\mathbf{u}^{1}-\mathbf{u}^{2}\right), \quad z=-d_{1},
$$

where the spring constant is

$$
\kappa=\frac{8}{\pi C l \beta}, \quad \beta=\frac{\lambda_{1}+2 \mu_{1}}{\mu_{1}\left(\lambda_{1}+\mu_{1}\right)}+\frac{\lambda_{2}+2 \mu_{2}}{\mu_{2}\left(\lambda_{2}+\mu_{2}\right)},
$$

and $C=2 l / a$ is relative density of cracks. It may be surprising that the spring constants are equal in the normal and tangential directions. However, it follows from the analysis in Golub and Boström [1], and it may be less surprising when it is noticed that the spring constant is independent of frequency. (And longitudinal and transverse waves behave in similar manners in many respects.) The same situation, with equal spring constants, also appear in the work of Boström and Wickham [9] for the case of partially closed cracks and is, furthermore, confirmed by comparisons with experiments.

The dispersion relations for thick layered plates with the spring boundary condition (19) between the layers and the boundary condition (2) or (3) on the external sides are easily derived and are not given here.

If the thickness of the plate, as well as the cracks, are small as compared to the characteristic wavelengths in the plate, then the problem can be further simplified in the context of plate theory. Here the approach of Boström et al. [18] is followed. Within this approach, the wave fields are expanded using Taylor power series expansions as

$$
\mathbf{u}^{1}(x, z)=\sum_{n=0}^{\infty} \mathbf{u}_{n}^{1}(x) z^{n} \quad \text { and } \quad \mathbf{u}^{2}(x, z)=\sum_{n=0}^{\infty} \mathbf{u}_{n}^{2}(x)\left(z+d_{1}\right)^{n}
$$

where $\mathbf{u}_{n}^{j}=\left(u_{x, n}^{j}, u_{z, n}^{j}\right)^{T}, j=1,2$. Substitution of these formulas into Eq. (1) leads to the recurrence relations

$$
\begin{aligned}
& \Delta_{1}^{j} u_{x, n}^{j}+(n+1)\left(\lambda_{j}+\mu_{j}\right) \frac{\partial u_{z, n+1}^{j}}{\partial x}+(n+2)(n+1) \mu_{j} u_{x, n+2}^{j}=0, \\
& \Delta_{2}^{j} u_{z, n}^{j}+(n+1)\left(\lambda_{j}+\mu_{j}\right) \frac{\partial u_{x, n+1}^{j}}{\partial x}+(n+2)(n+1)\left(\lambda_{j}+2 \mu_{j}\right) u_{z, n+2}^{j}=0, n=0,1,2, \ldots,
\end{aligned}
$$

where the wave operators are

$$
\Delta_{1}^{j}=\left(\left(\lambda_{j}+2 \mu_{j}\right) \frac{\partial^{2}}{\partial x^{2}}-\rho_{j} \frac{\partial^{2}}{\partial t^{2}}\right), \quad \Delta_{2}^{j}=\left(\mu_{j} \frac{\partial^{2}}{\partial x^{2}}-\rho_{j} \frac{\partial^{2}}{\partial t^{2}}\right), \quad j=1,2 .
$$


From these equations the expansion vector functions $\mathbf{u}_{n+2}^{j}$ can be expressed in terms of $\mathbf{u}_{n+1}^{j}$ and $\mathbf{u}_{n}^{j}$. The recurrence relations can thus be used to eliminate all the expansion functions except $\mathbf{u}_{0}^{j}$ and $\mathbf{u}_{1}^{j}$. Consequently, expansions (21) can be brought to a form with only the four expansion vector functions $\mathbf{u}_{0}^{j}$ and $\mathbf{u}_{1}^{j}, j=$ 1,2 . Their substitution into the spring boundary condition (19) imposes additional relations among them. In this way, one can further eliminate all the functions related to the second $(j=2)$ layer. Finally, boundary conditions $(2)$ give for the antisymmetric case (after elimination to a single equation) the scalar equation of bending motion of the plate

$$
A_{1} \frac{\partial^{4} u_{z, 0}^{1}}{\partial x^{4}}+B_{1} \frac{\partial^{4} u_{z, 0}^{1}}{\partial t^{2} x^{2}}+C_{1} \frac{\partial^{2} u_{z, 0}^{1}}{\partial t^{2}}+D_{1} \frac{\partial^{4} u_{z, 0}^{1}}{\partial t^{4}}+\frac{1}{\kappa}\left(E_{1} \frac{\partial^{4} u_{z, 0}^{1}}{\partial t^{2} x^{2}}+F_{1} \frac{\partial^{4} u_{z, 0}^{1}}{\partial t^{4}}\right)=0
$$

with coefficients

$$
\begin{gathered}
A_{1}=\frac{4}{3}\left[\mu_{2}\left(1-\gamma_{2}\right)\left(d_{2}^{3}-d_{1}^{3}\right)+\mu_{1} d_{1}^{3}\left(1-\gamma_{1}\right)\right], \quad C_{1}=d_{1} \rho_{1}+\left(d_{2}-d_{1}\right) \rho_{2}, \\
B_{1}=-\left[2\left(1-\gamma_{2}\right) d_{1}\left(d_{2}-d_{1}\right)^{2} \rho_{1}+\frac{4 \mu_{2}}{\mu_{1}}\left(1-\gamma_{2}\right) d_{1}^{2}\left(d_{2}-d_{1}\right) \rho_{1}+\frac{2}{3}\left(3-2 \gamma_{1}\right) d_{1}^{3} \rho_{1}\right. \\
\left.+\frac{4 \mu_{2}}{\mu_{1}}\left(1-\gamma_{2}\right) d_{1}\left(d_{2}-d_{1}\right)^{2} \rho_{2}+2 \gamma_{2} d_{1}\left(d_{2}-d_{1}\right)^{2} \rho_{2}+2 d_{1}^{2}\left(d_{2}-d_{1}\right) \rho_{2}+\frac{2}{3}\left(3-2 \gamma_{2}\right)\left(d_{2}-d_{1}\right)^{3} \rho_{2}\right], \\
D_{1}=\frac{1}{6 \mu_{1}}\left(3+\gamma_{1}\right) d_{1}^{3} \rho_{1}^{2}+\frac{1}{2 \mu_{1}}\left(3+\gamma_{1}\right) d_{1}^{2}\left(d_{2}-d_{1}\right) \rho_{1} \rho_{2}+\frac{1}{2 \mu_{2}}\left(1+\gamma_{2}\right) d_{1}\left(d_{2}-d_{1}\right)^{2} \rho_{1} \rho_{2} \\
+\frac{1}{6 \mu_{2}}\left(3+\gamma_{2}\right)\left(d_{2}-d_{1}\right)^{3} \rho_{2}^{2}+\frac{1}{\mu_{1}} d_{1}\left(d_{2}-d_{1}\right)^{2} \rho_{2}^{2}, \\
E_{1}=-4 \mu_{2}\left(1-\gamma_{2}\right)\left(d_{2}-d_{1}\right)\left(d_{1} \rho_{1}+d_{2} \rho_{2}\right), \quad F_{1}=2 d_{1}\left(d_{2}-d_{1}\right) \rho_{1} \rho_{2}+\left(d_{2}-d_{1}\right)^{2} \rho_{2}^{2},
\end{gathered}
$$

where

$$
\gamma_{1}=\frac{\mu_{1}}{\lambda_{1}+2 \mu_{1}}, \gamma_{2}=\frac{\mu_{2}}{\lambda_{2}+2 \mu_{2}}
$$

In the symmetric case boundary conditions (3) give the plate equation with respect to the longitudinal displacement $u_{x, 0}^{1}$

$$
A_{2} \frac{\partial^{2} u_{x, 0}^{1}}{\partial x^{2}}+B_{2} \frac{\partial^{2} u_{x, 0}^{1}}{\partial t^{2}}+\frac{1}{\kappa}\left(C_{2} \frac{\partial^{4} u_{x, 0}^{1}}{\partial x^{4}}+D_{2} \frac{\partial^{4} u_{x, 0}^{1}}{\partial t^{2} \partial x^{2}}+E_{2} \frac{\partial^{4} u_{x, 0}^{1}}{\partial t^{4}}\right)=0
$$

with

$$
A_{2}=4\left[\mu_{2}\left(1-\gamma_{2}\right)\left(d_{2}-d_{1}\right)+\mu_{1}\left(1-\gamma_{1}\right) d_{1}\right], \quad B_{2}=-\left[d_{1} \rho_{1}+\left(d_{2}-d_{1}\right) \rho_{2}\right],
$$




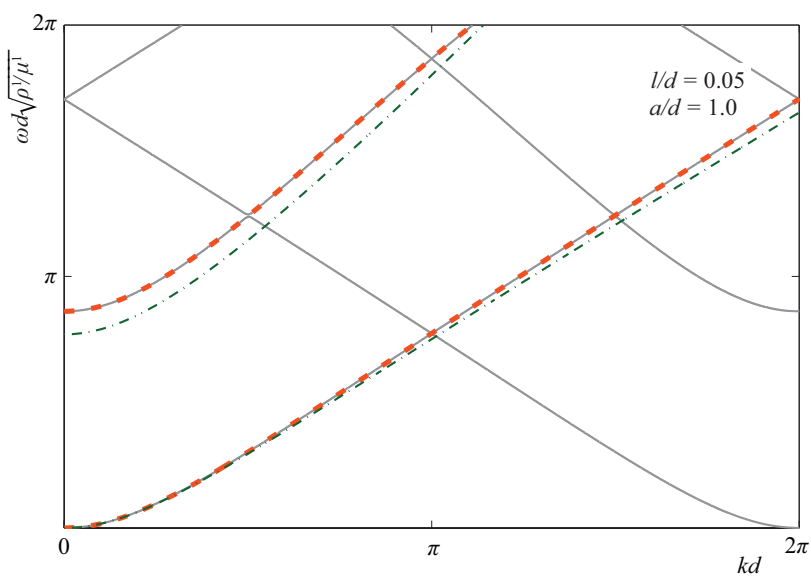

Figure 2. The wave numbers $k d$ of the antisymmetric modes as a function of frequency $\omega d \sqrt{\rho^{1} / \mu^{1}}$ for $l / d=0.05$ and $a / d=1.0$; solid lines: thick plate with periodic cracks, dotted lines: thick plate with spring boundary condition; dash-dotted lines: plate equation with spring boundary condition.

$$
\begin{aligned}
& C_{2}=-16 \mu_{1} \mu_{2}\left(1-\gamma_{1}\right)\left(1-\gamma_{2}\right) d_{1}\left(d_{2}-d_{1}\right), \quad E_{2}=-\left[2 d_{1}\left(d_{2}-d_{1}\right) \rho_{1} \rho_{2}+\left(d_{2}-d_{1}\right)^{2} \rho_{2}^{2}\right], \\
& D_{2}=4\left[\mu_{2}\left(1-\gamma_{2}\right) d_{1}\left(d_{2}-d_{1}\right) \rho_{1}+\mu_{2}\left(1-\gamma_{2}\right)\left(d_{2}-d_{1}\right)^{2} \rho_{2}+2 \mu_{1}\left(1-\gamma_{1}\right) d_{1}\left(d_{2}-d_{1}\right) \rho_{2}\right] .
\end{aligned}
$$

The first equation is written to the third order in thickness, the second one to second order, but it is straightforward to go to higher orders. For both cases it is straightforward to generalize to a plate in three dimensions by just changing all second order derivatives in $x$ to a sum of second order derivatives in $x$ and $y$ (or the 2D Laplacian).

In the antisymmetric (bending) equation the $A_{1}$ and $C_{1}$ terms are recognized as the Kirchhoff equation. Including also the $B_{1}$ and $D_{1}$ terms a Mindlin type of equation is obtained, although the coefficients are not the same. The last two terms that depend on the spring constant $\kappa$ reflect the presence of the interface cracks. These terms contain the same order of derivatives as the $B_{1}$ and $D_{1}$ terms. The quotient between the $B_{1}$ and $E_{1}$ terms is of the order of the relative density of cracks $C$ times a factor depending on $l / d$, where $d$ is the total plate thickness. The spring terms can thus be of the same order as the correction terms of Mindlin type to the Kirchhoff equation, although both $C$ and $l / d$ should be less than 1 .

In the symmetric (stretching) equation the first two terms in $A_{2}$ and $B_{2}$ describe usual in-plane motion. The other terms depend on the spring constant, and due to the higher order derivatives these terms will generally be small at low frequencies.

\section{Numerical examples}

In this section some numerical results are given to compare the approximations derived for the damaged layered plate with the exact solution for the thick plate with a periodic array of interface cracks as treated in Section 2. The first approximation is the thick plate with spring boundary condition (19). The second approximation is to use the plate equation (22) or (23) (taken to the order given), with the dispersion relations following directly from them. In this way both the spring boundary condition and the plate equations are checked.

The middle layer of the plate is made of aluminum $\left(\nu_{1}=0.35, \rho_{1}=2.7\right.$. $\left.10^{3} \mathrm{~kg} / \mathrm{m}^{3}, v_{s 1}=3110 \mathrm{~m} / \mathrm{s}\right)$ and of thickness $2 d_{1} / d=2 / 3$, while the top and 


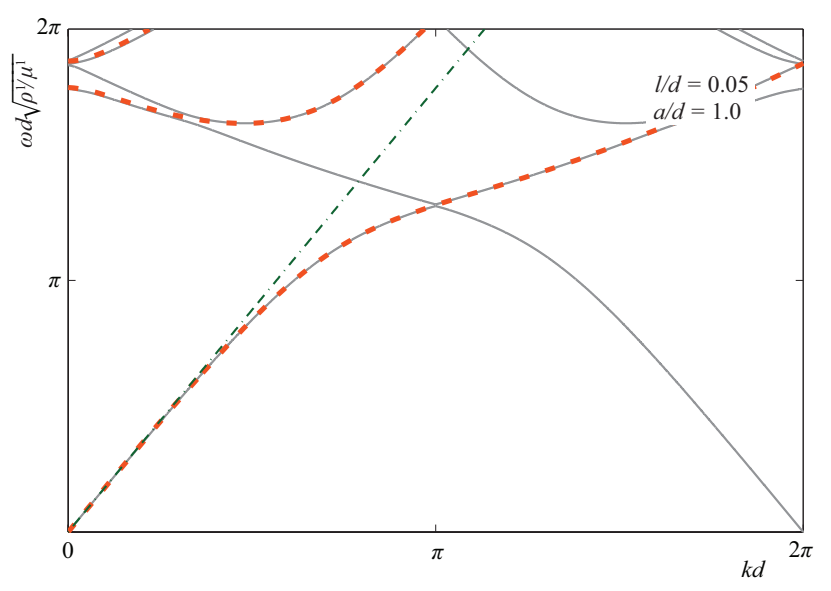

Figure 3. The same as in Fig. 2, but for symmetric modes.

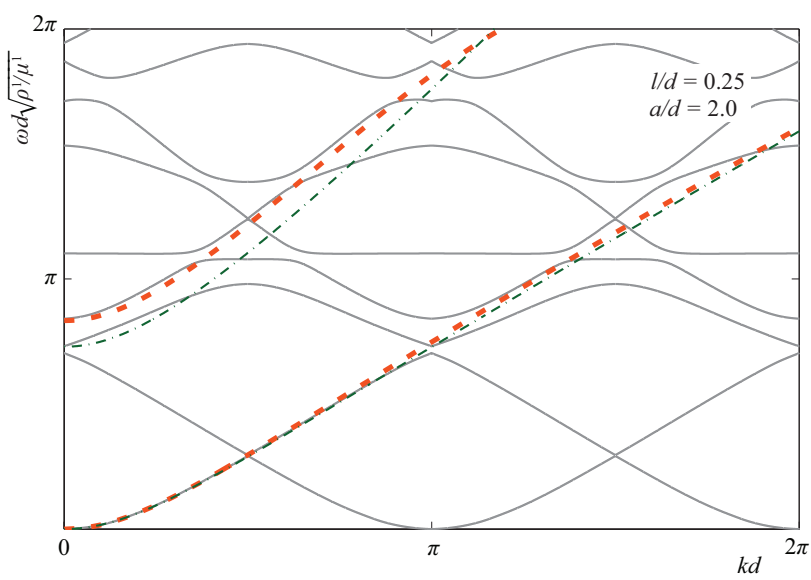

Figure 4. The same as in fig. 2 , but for $l / d=0.25$ and $a / d=2.0$.

bottom ones are of titanium $\left(\nu_{2}=0.3, \rho_{2}=4.4 \cdot 10^{3} \mathrm{~kg} / \mathrm{m}^{3}, v_{s 2}=3270 \mathrm{~m} / \mathrm{s}\right)$, with thickness $2\left(d_{2}-d_{1}\right) / d=1 / 3$.

Figures $2-7$ show the dimensionless wave number $k d$ as a function of the dimensionless frequency $\omega d \sqrt{\rho_{1} / \mu_{1}}$. Figures with even numbers show results for antisymmetric modes and figures with odd ones show results for symmetric modes. The corresponding crack sizes $(l / d=0.05,0.25$ and 0.5$)$ and spacings $(a / d=1$ and 2) are shown in the figures. The solid lines show the results for the thick plate with periodic interface cracks, i.e. the roots of dispersion equation (18), the dashed lines are the results for the thick plate with spring boundary condition (19), and the dash-dotted lines are the results for plate equation (22) or (23).

As the interface cracks form a periodic system, this gives a system of Bloch modes with periodicity in the $k d$ direction for the solid lines. Then, the dispersion curves obtained for the plate with spring boundary conditions (dashed lines) are typical for fundamental and higher-order guided waves propagating in elastic layered waveguides. For the most part they coincide or closely follow the ascending parts of the Bloch modes, whereas no guided waves may be associated with the descending ones. The approximate plate equations, in accordance with their asymptotic nature, describe only fundamental modes. That is why they give only two dispersion curves for antisymmetric modes (dash-dotted lines in Figs. 2, 4 and 6 ) and one (Figs. 3 and 5) or two (Fig. 7) curves in the symmetric case.

For the first antisymmetric and symmetric modes at low frequencies all three 


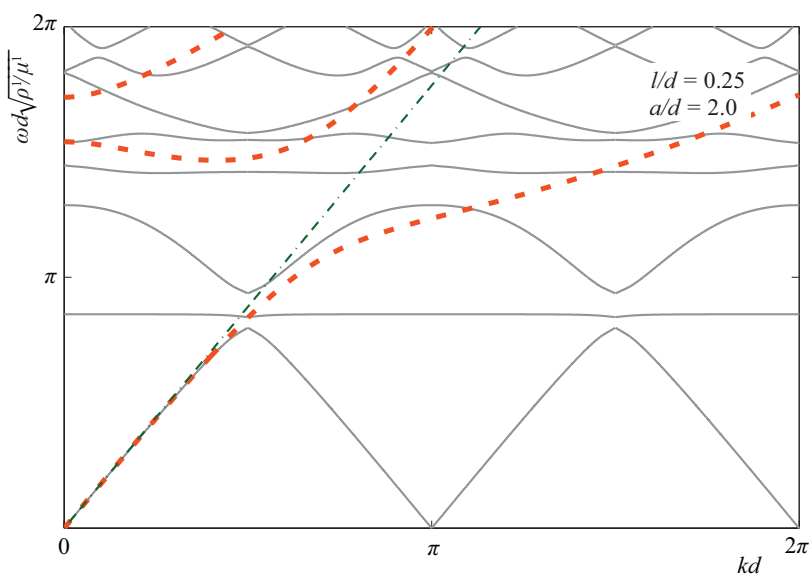

Figure 5. The same as in fig. 3, but for $l / d=0.25$ and $a / d=2.0$.

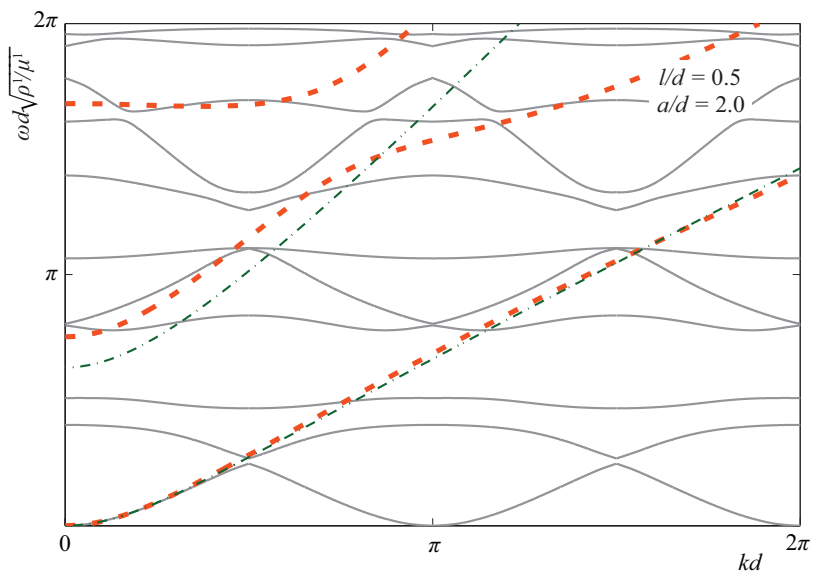

Figure 6. The same as in fig. 2 , but for $l / d=0.5$ and $a / d=2.0$.

theories agree very well, while for higher frequencies it is seen that the thick plate with the spring boundary condition performs better than the plate theory.

\section{Concluding remarks}

In-plane P-SV modes in a layered plate with periodic distribution of interface cracks have been investigated. The exact dispersion relation for a thick plate is compared to the case when the interface cracks are approximated by means of a distributed spring interface condition and also to plate equations incorporating this spring interface condition. At low frequencies the agreement is very good and this shows that the spring interface conditions can be used to model a periodic array of interface cracks. A model with a distribution of interface cracks is a plausible model for interface damage, and at low frequencies periodic and random distributions of cracks give very similar wave propagation characteristics. Therefore, the spring interface conditions should be a simple useful model for such damage. This model can then be useful in more complex situations, like a plate with damage along a finite part of the interface.

The situation considered here is only 2D, but there should be no principal obstacles for investigating 3D problems as well, in particular for the case with a rectangular interface crack. Also anisotropic media are of great practical interest 


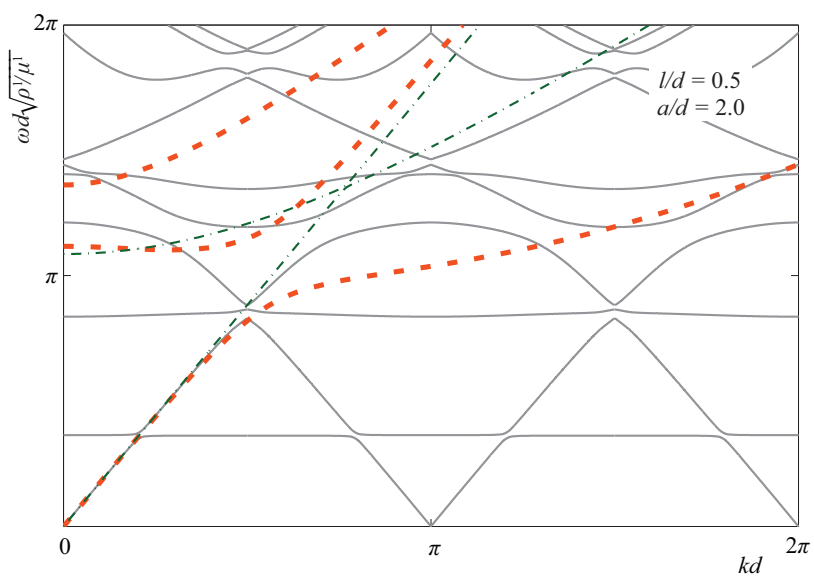

Figure 7. The same as in fig. 3 , but for $l / d=0.5$ and $a / d=2.0$.

as most composites can be modeled as anisotropic.

\section{Acknowledgements}

The authors are grateful to Dr. K. Mauritsson for fruitful discussions and valuable advices.

The work is supported by the Russian Ministry for Education and Science grant No. 2.1.1/10463 and by the Russian Foundation for Basic Research

\section{References}

[1] M. Golub and A. Boström, Interface damage modeled by spring boundary conditions for in-plane elastic waves, Wave Motion 48 (2011), pp. 105-115.

[2] A. Boström and O.V. Kvasha, Elastic SH wave propagation in a layered anisotropic plate with periodoc interface cracks: exact versus spring boundary conditions, J. Mech. Mater. Struct. 5 (2010), pp. 67-78.

[3] Ch. Zhang and D. Gross, On wave propagation in elastic solids with cracks, Computational Mechanics Publications, Southampton, UK, 1998.

[4] Y.C. Angel and J.D. Achenbach, Reflection and transmission of elastic waves by a periodic array of cracks, J. Appl. Mech. 52 (1985), pp. 33-41.

[5] Y. Mikata and J.D. Achenbach, Interaction of harmonic waves with a periodic array of inclined cracks, Wave Motion 10 (1988), pp. 59-72.

[6] J.M. Baik and R.B. Thompson, Ultrasonic scattering from imperfect interfaces: a quasi-static model, J. Nondestructive Eval. 4 (1984), pp. 177-196.

[7] S.I. Rokhlin and Y.J. Wang, Analysis of boundary conditions for elastic wave interaction with an interface between two solids, J. Acoust. Soc, Am. 89 (1991), pp. 503-515.

[8] S.I. Rokhlin and W. Huang, Ultrasonic wave interaction with a thin layer between two anisotropic solids, J. Acoust. Soc. Am. 92 (1992), pp. 1729-1742.

[9] A. Boström and G.R. Wickham, On the boundary conditions for ultrasonic transmission by partially closed cracks, J. Nondestructive Eval. 10 (1991), pp. 139-149.

[10] S. Nakagawa, K.T. Nihei and L.R. Myer, Plane wave solution for elastic wave scattering by a heterogeneous fracture, J. Acoust. Soc. Am. 115 (2004), pp. 2761-2772.

[11] C. Pecorari, Spring boundary model for a partially closed crack, Int. J. Eng. Sci. 46 (2008), pp. $182-188$.

[12] J.D. Achenbach and Ch. Zhang, Reflection and transmission of ultrasound by a region of damaged material, J. Nondestructive Eval. 9 (1990), pp. 71-79.

[13] J.D. Achenbach, M. Kitahara, Y. Mikata and D.A. Sotiropoulos, Reflection and transmission of plane waves by a layer of compact inhomogeneities, Pure Appl. Geophysics 128 (1988), pp. 101-118.

[14] A. Yariv and P. Yeh, Optical waves in crystals: propagation and control of laser radiation, Wiley, New York, 1984.

[15] V.A. Babeshko, E.V. Glushkov, and J.F. Zinchenko, The dynamics of inhomogeneous linearly-elastic bodies, Nauka, Moscow, 1989 (in Russian).

[16] E. Glushkov, N. Glushkova, M. Golub and A. Boström, Natural resonance frequencies, wave blocking, and energy localization in an elastic half-space and waveguide with a crack, J. Acoust. Soc. Am. 119 (2006), pp. 3589-3598.

[17] A. Boström and M. Golub, Elastic SH wave propagation in a layered anisotropic plate with interface damage modeled by spring boundary conditions, Q. J. Mech. Appl. Math. 62 (2009, pp. 39-52. 
[18] A. Boström, G. Johansson, and P. Olsson, On the rational derivation of a hierarchy of dynamic equations for a homogeneous isotropic elastic plate, Int. J. Solids Struct. 38 (2001), pp. 2487-2501. 